\title{
The Effects of Walking, Feedback and Control Method on Pressure-Based Interaction
}

\author{
Graham Wilson, Stephen A. Brewster, Martin Halvey, Andrew Crossan \& Craig Stewart \\ Glasgow Interactive Systems Group, School of Computing Science \\ University of Glasgow, Glasgow G12 8QQ \\ gawilson@dcs.gla.ac.uk, \{First.Last\}@glasgow.ac.uk \\ www.dcs.gla.ac.uk/ stephen
}

\begin{abstract}
This paper presents a study looking into the effects of walking and the use of visual and audio feedback on the application of pressure for linear targeting. Positional and Ratebased control methods are compared in order to determine which allows for more stable and accurate selections, both while sitting and mobile. Results suggest that Rate-based control is superior for both mobile (walking) and static (sitting) linear targeting, and that mobility significantly increases errors, selection time and subjective workload. The use of only audio feedback significantly increased errors and task time for Positional control and static Rate-based control, but not mobile Rate-based control. Despite this, the results still suggest that audio control of pressure interaction while walking is highly accurate and usable.
\end{abstract}

\section{Author Keywords}

Pressure input, non-visual feedback, mobile interaction.

\section{ACM Classification Keywords}

H5.2. User Interfaces: Haptic IO

\section{General Terms}

Design, Human Factors.

\section{INTRODUCTION}

Pressure/force input sits as a natural augmentation of the increasingly common touch interaction paradigms among mobile devices: those based on interaction through touchscreens, accelerometers and gestures. Pressure input also brings with it several benefits for mobile interaction such as real-time and fine-grained control over a single-axis input including media traversal (rewind/fast-forward), zooming and scrolling, replacing potentially inaccurate, repetitive and screen-occluding 'flick' or 'pinch' touchscreen gestures. It can add false physicality to virtual buttons, requiring a degree of force to activate, rather than solely a touch event, which may be beneficial for less desired options such as file deletion. An extension of this is giving virtual surfaces/objects physical properties such as texture, weight or friction through the addition of vibrotactile feedback [6].

\footnotetext{
Permission to make digital or hard copies of all or part of this work for personal or classroom use is granted without fee provided that copies are not made or distributed for profit or commercial advantage and that copies bear this notice and the full citation on the first page. To copy otherwise, or republish, to post on servers or to redistribute to lists, requires prior specific permission and/or a fee.
}

MobileHCI 2011, Aug 30-Sept 2, 2011, Stockholm, Sweden. Copyright 2011 ACM 978-1-4503-0541-9/11/08-09....\$10.00.
As a final example more relevant to the research presented here, pressure can be used for menu interaction using a form of linear targeting. A body of research has shown pressure-based linear targeting (shortened here to PBLT) to be highly accurate using up to 8-10 targets/menu items while stationary and using visual feedback [3, 9, 11, 12], but much less work has looked at control while walking and using audio feedback. There are two related benefits that this kind of input can bring to interaction with mobile devices while the user is in motion. Firstly, it removes the need for accurate pointing on touchscreen menus when the hands and device are moving, and secondly, through the use of audio feedback, it allows for eyes-free interaction.

As an illustration of a potentially beneficial implementation of PBLT we will use listening to music on a touchscreen device while walking. In order to, for example, pause or change tracks the user must have the device in their hand in front of them and be looking at it to press the correct virtual button. A not uncommon process may involve: 1) taking the device out of a pocket, 2) waking the device screen, 3) swiping or entering a PIN to unlock the screen before 4) pressing the desired virtual button (many devices may have covers or sleeves which must also be removed). This is time-consuming and takes visual attention away from the environment. If a device was pressure sensitive, for example with sensors lining the sides of the device, the user could reach into the pocket and squeeze the device to interact with the mp3 player menu through linear targeting.

This paper expands the research from Wilson et al. [16] by looking at the control of PBLT while the user is walking. In order to determine the feasibility of eyes-free interaction, we compared both visual and audio-only control. Within past PBLT research, nearly all studies have used the same control/interaction method, known as Positional control. However, results from pressure-based shape translation [13] and targeting through head tilt [4] have suggested that a velocity or Rate-based control may allow for better control of pressure or linear targeting (respectively), especially when the user is mobile. Therefore, in this paper we present two parts of one study looking at pressure input on mobile devices: one looking at how walking affects our ability to control pressure-based input and which control method allows for better control; and a second testing whether the same mobile interaction can be carried out with only audio feedback. 


\section{BACKGROUND}

\section{Pressure Input and Linear Targeting}

In pressure-based linear targeting (PBLT) the pressure space (the total amount of pressure the sensor can detect, or a limit set by the implementation) is divided into a number of bins or levels of equal width (in Newton's or sensor values) and the user applies pressure to a target level of force (see Figure 1). Making the target levels thinner gives an indication of how precisely users can apply pressure and how many levels can feasibly be used in an interaction (e.g. menu size, zoom levels, paint brush thickness).

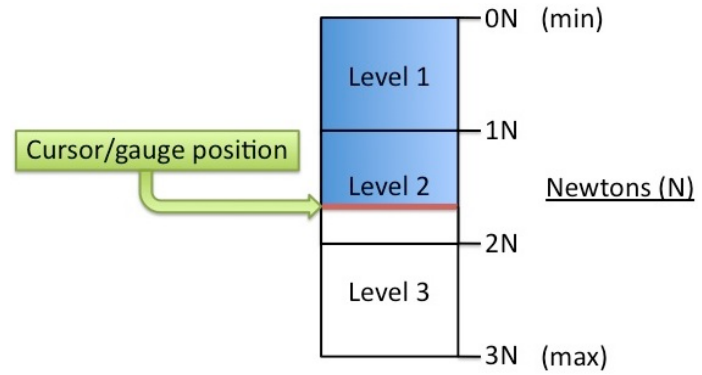

Figure 1: Example linear targeting implementation. Cursor position is dictated by applied force (Positional control): applying more force moves gauge further down.

Almost all PBLT studies have used Positional control of input, illustrated in Figure 1, where the gauge fills (or a cursor moves through the levels) to a position relative to the amount of pressure applied. Releasing pressure moves the gauge/cursor back towards the beginning. The conclusions from PBLT research regarding the maximum number of useable levels have varied, depending on the hardware, software treatments, analog-to-digital conversion, or size of pressure space used, but there is a general consensus that, when using Positional control, accuracy begins to decline sharply above 8 or 10 levels $[3,9,11,12]$. PBLT research run on mobile devices while the user remains stationary has come to similar conclusions $[9,16]$.

Research looking at pressure input on mobile phones has also focused on linear targeting in the guise of text entry. Both McCallum et al. [8] and Brewster \& Hughes [2] looked at using pressure for text entry but in different ways. McCallum et al. used a pressure-sensitive phone keypad and found that it allowed for a higher Words Per Minute value than traditional MultiTap input. Brewster \& Hughes are the only authors to look at pressure input for linear targeting while the user is walking. They displayed a QWERTY keyboard on the resistive screen of the Nokia N800 and used two pressure levels for lowercase and uppercase characters respectively, thereby avoiding repeated trips to press the shift key. Being mobile did not significantly increase mean character input time but did significantly increase errors. The use of the Dwell selection technique somewhat mitigated these effects, however. This technique involves maintaining the target level of pressure for a set time (e.g. 1 second) to confirm selection.
All the above studies used Positional control for the interaction. An alternative control method would be Rate-based control, where the velocity of an interaction element, in this case the speed of the cursor or gauge's movement through the interaction space, is controlled by the amount of pressure being applied. Using an interaction like that in Figure 1 , pressing lightly on the sensor would move or increase the gauge slowly to the right, further along the interaction space. Pressing harder would increase the gauge faster. Shi et al. [13] found that Rate-based control allowed for more precise, faster and less mentally/physically demanding control of pressure-based shape translation (rotation). Outside of pressure interaction, but remaining within linear targeting, Crossan et al. [4] found that Rate-based control of cursor movement through head tilting produced more accurate selections than Positional control while the user was walking (with Positional control being faster and more accurate when the user was standing still).

Aside from Brewster \& Hughes [2], no other work has studied the effect of mobility on pressure input, and the aforementioned study only used 2 levels of pressure, whereas the stationary studies went up as high as 12 . Therefore it is necessary to ascertain whether the same interactions can be carried out while walking. As Rate-based control may allow for more accurate or stable control for mobile interaction it was decided to compare performance using both control methods while the user was both sitting and walking.

\section{Mobile and Audio Interaction}

As suggested above, pressure input lends itself well to an augmentation of current gestural and multitouch interfaces dominating mobile devices. But an important faculty of mobile devices is the ability to interact while actually onthe-move. Walking can make interacting with mobile devices more challenging compared to interaction while sitting or standing, especially if the task involves accurate targeting. Interacting while walking can increase task time $[1,4,7]$ and reduce accuracy $[4,5]$, while inadvertent movement caused by specific phases in a users gait has been shown to interfere with deliberate movement and so decrease accuracy during touchscreen interaction [5]. The use of audio feedback has been shown to improve mobile interactions, both in conjunction with visual feedback [1] and during audio-only interaction [8].

The vast majority of HCI research on pressure, and therefore the vast majority of its successes, has been based on providing visual feedback during interaction. The use of audio feedback during pressure interaction has achieved mixed success. Some users report appreciating audio feedback added to a visual interaction [10]. Audio feedback elicited faster response time than tactile feedback during simultaneous application of pressure using 3 fingers [15]. However linear targeting using only audio feedback and 3 levels of pressure was significantly less accurate than visual or tactile (or multimodal tactile and audio) interaction with a phone-FSR 'sandwich' [14]. Wilson et al. [16] had users 
acquiring 4 to 10 levels with 95-63\% accuracy (respectively) using only audio feedback while seated at a desk.

Therefore we wanted to investigate whether an audio-only version of the linear targeting interaction is useable while both sitting and walking. This would enable eyes-free interaction with mobile devices, specifically touchscreen devices that cannot be operated 'in-pocket' due to their featureless input surface. In an attempt to achieve this we ran a controlled study split into two sessions. The first session compared Positional and Rate-based control of a visual feedback-only linear targeting task while the user was sitting in a chair and walking a set route indoors. From this study we could identify the effect of mobility on control of pressure and also identify which of the control methods allowed for fastest or most accurate/stable control while walking. Then, in session 2 we would use this control method and have users interacting with only audio feedback while both sitting and walking. From this session we would be able to determine the effect of feedback on the control of pressure by comparing performances from session 1 and 2 .

\section{SESSION 1: MOBILITY \& CONTROL}

\section{Apparatus}

The experimental software ran on a Samsung Q1 UMPC (see Figure 2). An Interlinks Electronics force-sensing resistor (FSR) model 402 (also Figure 2) was connected to the Q1 over USB via an SAMH Engineering SK7-ExtGPIO01 input/output module, which handled A-to-D conversion and sensor linearization [14]. The FSR was attached to the front bezel of the Q1 on the same side as the user's dominant hand. This same apparatus was used for both sessions.

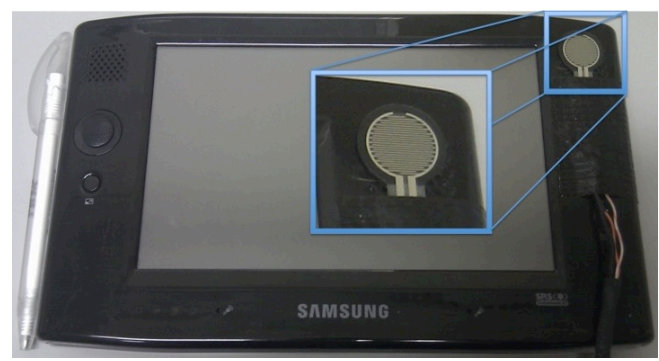

Figure 2: Samsung model Q1 with FSR attached (top right).

\section{Experimental Task}

The experimental task was a vertical linear targeting application, the same used by Wilson et al. [16], a variation on a common implementation $[3,9,11]$. The same task was used for both session 1 and 2 . A pressure space of approximately $4 \mathrm{~N}$ was divided into $4,6,8$ or 10 equal-sized bins/levels visualized on-screen as a vertical menu of as many menu items, running from top-to-bottom and measuring 200x400 pixels (39.6x79.2mm; see Figure 3). Each menu item had a label commonly used in many desktop and mobile applications, so as to give the task a semblance of real world use. Each trial involved selecting a single menu item: the participant had to move a square green cursor down the left side of the menu using pressure (see Control Methods below), starting at the top, so that it was within the boundaries of the target item before selecting it via a 1 -second $D$ well technique. Dwell has been shown to be most accurate in linear targeting, although it suffers longer overall targeting time due to the imposed dwell duration $[2,11]$.

\section{Control Methods}

Positional Control

In this method the position of the cursor in the menu is dictated by how hard the participant presses on the FSR (see Figure 1). This interaction is similar to pressing against a relatively loose spring as more force is required to move the cursor further down, but releasing pressure from the FSR results in the cursor 'springing' back up to the top. As bidirectional movement was possible, pressing too hard and overshooting a target could be fixed by alleviating a degree of pressure so the cursor moves back up the menu. As the menu and task were designed to have a degree of real-world relevance, it was impossible to move the cursor past the bottom of the menu. Target selection was achieved by maintaining a level of pressure that held the cursor within the target item for 1 second.
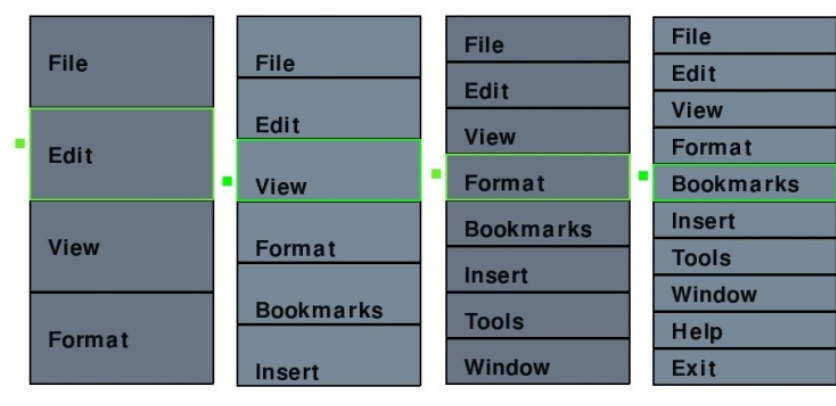

Figure 3: Menu layouts for the 4, 6, 8 \& 10 item menu sizes with relative target widths.

Rate-based Control

In this method the velocity of the cursor's downward motion is dictated by how hard the participant presses on the FSR, with no pressure bringing it to a halt. This interaction is similar to pushing an object along a smooth surface: how hard you push it dictates how fast it moves, and stopping pushing stops the objects movement. 'Velocity' in this case refers to the number of pixels (or millimeters) the cursor moves every cycle of the experimental software, which was approximately every $0.03 \mathrm{sec}$. Pilot testing led to the adoption of a maximum speed of 10 pixels $(2 \mathrm{~mm})$ per cycle $(330$ pixels $/ 66 \mathrm{~mm}$ per second; see Table 1$)$. Initially a maximum speed of 20 pixels $(4 \mathrm{~mm})$ was chosen, balancing speed and control, but this was found to be too fast for accurate control when using audio feedback. This design only allowed for downward motion of the cursor. Although a second FSR could have been used to allow for upward motion (in the case of overshooting a target), the Positional control method only utilized one sensor, so it was decided to use only one for Rate-based control also, to keep the interactions as similar as possible (even though Positional control allows for bi-directional movement). Therefore, if the participant overshot a target, they could push the cursor past 
the bottom of the menu and it would 'loop' back to the top of the menu and start again. Stopping the cursor within the target item (by lifting off the FSR) and leaving it stationary for 1 second achieved target selection.

\begin{tabular}{|c|c|c|c|c|c|c|c|c|c|c|c|}
\hline $\begin{array}{c}\text { Approx. } \\
\text { Pressure (N) }\end{array}$ & 0 & 0.2 & 0.4 & 0.6 & 0.8 & 1.0 & 1.2 & 1.4 & 1.6 & 1.8 & 2.0 \\
\hline $\begin{array}{c}\text { Speed pix/sec } \\
(\mathbf{m m} / \mathbf{s e c})\end{array}$ & 0 & $\begin{array}{c}33 \\
(6.6)\end{array}$ & $\begin{array}{c}66 \\
(13.2)\end{array}$ & $\begin{array}{c}99 \\
(19.8)\end{array}$ & $\begin{array}{c}132 \\
(26.4)\end{array}$ & $\begin{array}{c}165 \\
(33)\end{array}$ & $\begin{array}{c}198 \\
(39.6)\end{array}$ & $\begin{array}{c}231 \\
(46.2)\end{array}$ & $\begin{array}{c}264 \\
(52.8)\end{array}$ & $\begin{array}{c}297 \\
(59.4)\end{array}$ & $\begin{array}{c}330 \\
(66)\end{array}$ \\
\hline
\end{tabular}

Table 1: Rate-based condition speeds in pixels and millimeters per second, based on pressure input in Newton's $(N)$.

\section{Mobility}

During the static condition, participants were sat in a padded office chair holding the UMPC in both hands. They were allowed to rest their arms on either their legs/knees or a desk in front of them to provide stability, but could not rest the device or their wrists while interacting. The mobile condition used a similar design to Crossan et al. [4] as it requires divided visual attention between task and navigation. Participants were asked to walk in a $4 \mathrm{~m} \times 3 \mathrm{~m}$ figureof-eight route indoors while they interacted with the device. The route was marked by four pieces of paper, one at each corner of the $4 \mathrm{~m} \times 3 \mathrm{~m}$ rectangle and users held the device in both hands with no further support.

\section{Participants \& Experimental Procedure}

Fourteen participants ( 11 male, 3 female) aged between 17 and 30 years old (mean 22.8) took part in the evaluation, all of whom were from within the University. Thirteen were right-handed and all were paid $£ 20$ for participation in both sessions of the study.

The experiment was a 2 x 2 design (mobility x control) so that participants completed two static and two mobile conditions, using each of the control methods: StaticPositional, Static-Rate, Mobile-Positional and Mobile-Rate. The order of these four conditions was counterbalanced to avoid order effects. Within each condition every menu item from all four of the menu sizes (4, 6, 8 and 10 items) was to be selected twice. The presentation order of menu sizes was randomized, and all targets within that menu were presented in a random order. At the start of each trial, the target item was highlighted in green for one second before returning to the same grey as the menu and each condition began with 10 practice selections. Participants completed a NASA TLX workload estimation form after each condition.

\section{Variables \& Measures}

There were three Independent Variables: Control Method (Positional, Rate-based), Mobility (Sitting, Walking) and Menu size (4, 6, 8 or 10 items).

Dependent Variables were: Errors (ER; whether the correct menu item was selected); Movement Time (MT; time between first non- 0 pressure value and selection, be that correct or incorrect); Number of Crossings (NC; the number of times the cursor crossed either of a target item's boundaries during Positional control); Loops (the number of overshot/looped targets in Rate-based control); Nudges (the number of discrete presses on the FSR to 'nudge' cursor along during Rate-based); Workload measured via the NASA TLX.

$\mathrm{NC}$ has been used as an indication of control, as a lower number suggests more stable and precise application of pressure, however it applies only to Positional control. A somewhat similar measure, here called Loops, was used for Rate-based conditions and measured the number of overshot attempts. A final objective measure recorded during Rate-based conditions was what we called Nudges: the number of press-release cycles the user employs to move the cursor, essentially 'nudging' or 'shunting' the cursor along as a sort of searching behaviour. This may indicate lower confidence in control over the input.

\section{RESULTS - SESSION 1}

When comparing between conditions, the data for every target selected within that condition was used in the analysis. However, as there were an uneven number of targets in the different menu sizes, when analyzing the potential effect of Menu Size on the variables, but only the effect of Menu Size, we only used 4 targets from each menu size, those identified by Ramos et al. [11] as having within them a set equal distance from 0 pressure. This choice means that, for example, " $25 \%$ errors" means the same number of errors for a 4-item menu and a 10-item menu (i.e. 1 incorrect selection), so allows for more even comparison. However it does raise the problem that incorrect selections from out with these 4 targets will not be counted when comparing the effect of Menu Size.

As some of the data did not fit a normal distribution, we used non-parametric analyses specifically Wilcoxon $T$ test for pairwise comparisons and the Friedman test for nonparametric ANOVA equivalent. Although the use of nonparametric tests increases the validity of results gained from non-normal data, they are limited in their inability to examine interaction effects. For normally distributed data, ANOVA was used.

\section{Errors}

A Wilcoxon pairwise comparison showed a significant effect of mobility on number of errors $(T=682.50, \mathrm{p}<.01)$, as walking (mean $=3.1 \%)$ produced more errors than sitting (mean $=1.7 \%)$. There was no effect of control method on errors $(T=1242.50, \mathrm{p}>.05)$ as both had ER of $2.4 \%$. Comparing conditions, Wilcoxon $T$ pairwise comparisons showed a significant difference between the Static-Rate and the Mobile-Rate conditions $(T=170, \mathrm{p}<.05)$. All other comparisons were not significant $(\mathrm{p}>.05)$. Error rates for the four conditions (see Figure 4) were: $1.8 \%$ for StaticPositional ( $\mathrm{SD}=0.13), 1.5 \%$ for Static-Rate $(\mathrm{SD}=0.12)$, $2.9 \%$ for Mobile-Positional $(\mathrm{SD}=0.17)$ and $3.2 \%$ for $\mathrm{Mo}$ bile-Rate $(\mathrm{SD}=0.18)$.

Friedman's Test showed a significant effect of Menu Size on $\operatorname{ER}\left(\chi^{2}(2)=9.867, \mathrm{p}<.05\right)$. Further Wilcoxon pairwise comparisons showed that ER for the 10 item menu was sig- 
nificantly higher than both the 4 item menu $(T=50.0, \mathrm{p}<.05)$ and the 8 item menu $(T=27.0, \mathrm{p}<.01)$. Mean ER values for each menu size were $1.2 \%, 2.4 \%, 0.7 \%$ and $3.4 \%$ for $4,6,8$ and 10 item menus respectively.

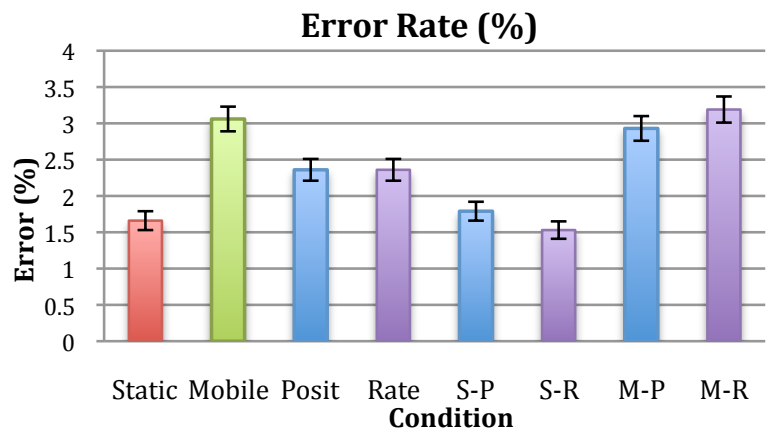

Figure 4: Mean error rates and SD for session 1 conditions: Static (S), Mobile (M), Positional (P) and Rate-based (R).

\section{Movement Time}

Both mobility $(T=472356.5, \mathrm{p}<.001)$ and control method ( $T=266186.5, \mathrm{p}<.001)$ significantly affected task time, with Rate-based control (mean $=2.29 \mathrm{~s}$ ) allowing for faster selections than Positional control (mean $=3.37 \mathrm{~s}$ ) and walking (mean=3.11s) causing slower selections than sitting (mean=2.55s). Wilcoxon $T$ comparisons found that all conditions were significantly different from each other $(\mathrm{p}<.001)$, with the exception of Static-Rate vs. Mobile-Rate ( $>$.05). Mean movement times for each condition (including the one-second Dwell time) were 2.85s (StaticPositional), 2.24s (Static-Rate), 3.88s (Mobile-Positional) and 2.34s (Mobile Rate; see Figure 5).

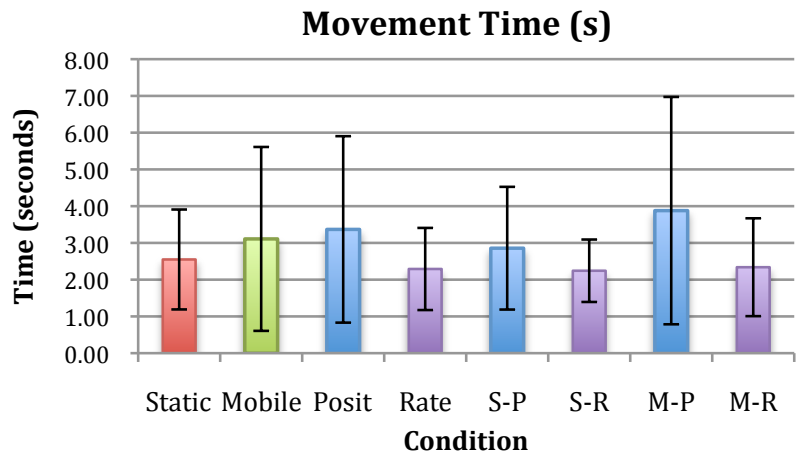

Figure 5: Mean target selection times with SD for each condition during session 1.

Menu size also had a significant effect on MT $\left(\chi^{2}\right.$ $(3)=307.861, p<.001)$. Wilcoxon comparisons showed that all menu sizes differed from each other significantly $(\mathrm{p}<.001)$, with mean MT of $2.14 \mathrm{~s}, 2.54 \mathrm{~s}, 2.94$ and $3.55 \mathrm{~s}$ for 4, 6, 8 and 10 item menus respectively.

\section{Number of Crossings/Loops}

NC only applies to the Positional control method and so only Static-Positional and Mobile-Positional were compared. Wilcoxon pairwise comparison showed that mobility had a significant effect on the number of crossings $(T=170$, $\mathrm{p}<.05)$ with mobile selections resulting in more crossings (mean=6.25) per target than static selections (mean=3.46). Menu size significantly affected NC (Friedman's $\chi^{2}$ $(3)=181.69, \mathrm{p}<.001)$ with the NC for each menu size differing significantly from every other one (Wilcoxon $\mathrm{p}<.001$ ). Mean NC was $1.71,3.56,5.19$ and 7.69 for $4,6,8$ and 10 item menus respectively.

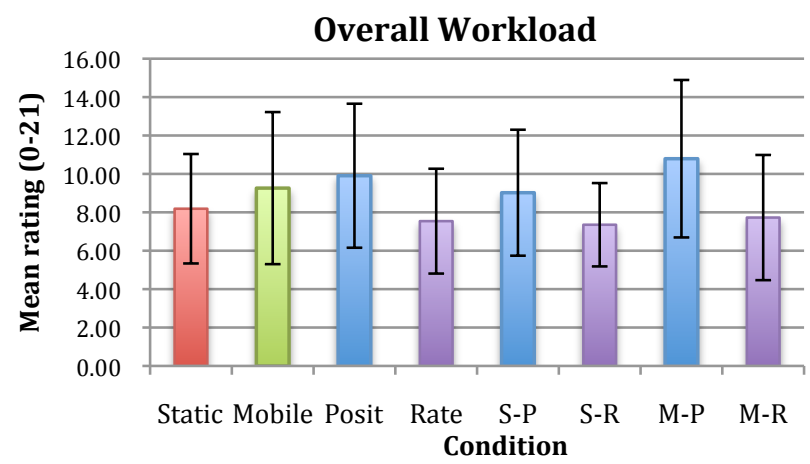

Figure 6: Mean overall subjective workload ratings and SD for each condition in session 1.

Loops only applied to the Static-Rate and Mobile-Rate conditions and there was a significant effect of mobility found on the number of overshoots (Wilcoxon $T=39.50, \mathrm{p}<.05$ ) with mobile selections producing significantly more overshoots per selection (mean=0.046) than static selections (mean=0.012). Menu size also had a significant effect on number of Loops (Friedman's $\chi^{2}(3)=12.789$, p<.01). Wilcoxon comparisons show that the 8 item menu produced significantly more Loops (mean $=0.06)$ per trial than both the 4 item menu (mean=0.01; $T=9.5, \mathrm{p}<.05)$ and the 6 item menu (mean $=0.00 ; T=4.5, \mathrm{p}<.01)$, and the 10 item menu (mean $=0.04)$ also produced significantly more Loops per trial than the 6 item menu $(T=4.0, \mathrm{p}<.05)$.

\section{Subjective Workload}

Repeated-measures ANOVA showed that being mobile significantly increased overall subjective workload compared to sitting $\left(\mathrm{F}_{1,13}=16.103, \mathrm{p}<.05\right.$; see Figure 6$)$ and that Positional control elicited significantly higher overall workload $\left(\mathrm{F}_{1,13}=78.381, \mathrm{p}<.01\right)$ than Rate-based control.

\section{Control Tactics}

The average number of nudges across both Static-Rate and Mobile-Rate was 0.22 nudges per selection. A Wilcoxon pairwise comparison showed a significant effect of mobility on the number of nudges $(T=1944, \mathrm{p}<.001)$ with mobile selections eliciting more nudges per selection (mean $=0.31$ ) than static selections (mean=0.13). Friedman's Test showed that Menu Size also significantly affected the number of nudges $\left(\chi^{2}(3)=51.209, \mathrm{p}<.001\right)$. All menu sizes differed significantly from each other $(\mathrm{p}<.001)$ except for 4 vs. 6 items and 8 vs. 10 items, which were not significantly different $(\mathrm{p}>.05)$. Mean number of nudges for each menu size was $0.08,0.08,0.37$ and 0.35 for $4,6,8$ and 10 item menus respectively. 


\section{INITIAL DISCUSSION \& CONCLUSIONS}

Walking had a big impact on user performance, producing more errors and taking, on average, one second longer per selection. It also greatly increased mental/physical workload levels. Although NC and Loops are not correlate measures, the higher values produced when walking indicate a lower degree of control during mobile selections. Interestingly, mobility appears to have a smaller impact on Rate-based selection time than on Positional selection time, possibly because the influence of unintended changes in input due to bodily movement is stronger for Positional control and so the participants had more difficulty 'homing in' on targets, or difficulty stopping the cursor in time. Walking increased average Positional selection time by $1.02 \mathrm{~s}$ but only increased it by $0.1 \mathrm{~s}$ under Rate-based control. Therefore use of Rate-based input may mitigate the negative effects of mobility to a degree.

The results from the first session strongly suggested that Rate-based input allows for superior control of pressurebased linear targeting compared to Positional input for both static and mobile interaction. Although both control methods enjoyed equal accuracy, Rate-based selections were significantly faster when both sitting and walking and were rated as significantly less mentally and physically demanding. Therefore this control method appears to be better than the standard method used in linear targeting research, with mobile Rate-based selections even being faster than static Positional ones.

As has been found in many other PBLT studies, menu size (thus target size) also significantly affected performance, with generally higher ER, MT, NC, Loops and Nudges occurring as the size of targets got smaller (i.e. the number of menu items increased), however ER and Loops did not increase smoothly. The low number of Nudges both overall and even when walking suggests that participants did not engage in 'shunting' or searching behaviour during Ratebased control, even though mobility produced a significantly greater number. Looking at pressure profiles also shows that many users maintained a set speed from start to finish and simply lifted their thumb as soon as the cursor was in the target item.

From these results it appears that mobility negatively influences pressure-based linear targeting but that Rate-based control mitigates these effects to an extent and so is best suited to mobile interaction. Therefore the Rate-based method was chosen for use during the second part of the experiment, which investigated whether users were able to interact with this application using only audio feedback.

\section{SESSION 2: MOBILITY \& FEEDBACK Experimental Design}

Thirteen of the same fourteen participants (11 male, 2 female) took part in the second session of the evaluation. One participant was unable to take part in session 2. The second session took place 4-6 weeks after session one.
The first condition that all participants engaged in was a walking condition using the Rate-based control with visual feedback, consisting of half as many selections as in other normal conditions. The purpose of this condition was to allow participants to familiarise themselves with both menu layouts and the cursor behaviour after the long break, so they were explicitly told to try and remember the layout/order of labels as best as they could during this time. Wilson et al. [17] found a lack of familiarity of menu layout lead to poorer performance in similar experiments.

After this familiarisation, the main study consisted of three audio-only conditions, presented in a counterbalanced order: Static-Rate-Audio (SRA), Mobile-Rate-Audio (MRA) and Mobile-Positional-Audio (MPA). Although Positional control was identified as resulting in poorer control and higher workload during session one, MPA was also included in this session to investigate whether our conclusions about Rate-based superiority for mobile non-visual interaction were reliable. The task was identical with every target from all four menu sizes being selected twice at random and in counterbalanced order after 10 practice selections. Audio feedback was presented to participants through stereo headphones connected to the UMPC. Participants completed a NASA TLX workload estimation form after each condition. The same variables measured during session one were measured during session 2. Independent variables were Condition (SRA, MRA and MPA) and Menu size (4, 6, 8 and 10 items).

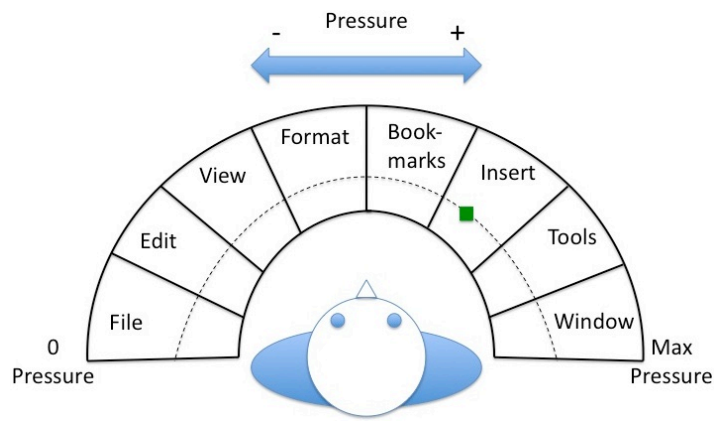

Figure 7: Illustration of audio feedback with menu laid out in 1D spatialised audio along the horizontal axis.

\section{Audio Feedback Design}

The design used here is very similar to that used by Wilson et al. [17]. In this design, the menu was effectively turned onto its left side so that it ran from left-to-right instead of top-to-bottom. The feedback was then panned along the egocentric horizontal axis, starting from the left (see Figure 7), so that it appeared to be laid out around the front of the user. The cursor moved along the menu based on pressure in exactly the same way as in the visual condition, only this time moved invisibly from left-to-right. Audio feedback consisted of each of the following for all menu items:

- Item label spoken in synthetic speech (i.e. "File")

- Unique musical tone

- 'Central tone': octave of unique tone 
When the cursor entered an item, from either direction, that item's label was spoken in synthetic speech. Each item also had a unique (ascending left-to-right) musical note that played for as long as the cursor was within that item. Finally, as users were reported to aim for the centre of targets during PBLT [9] a 'central tone' was played when the cursor was in the middle $1 / 3$ of an item. This tone was the same as the unique tone only one octave higher. All of these sounds were unique to each individual item and were played in stereo in the egocentric horizontal spatial location around the head relative to that items location in the menu. For example File would always be heard on the far left and the last item on the far right; for an 8-item menu "insert" would be heard just right-of-centre (see Figure 7).

The Rate-based audio conditions used the same design, only with the addition of one more cue to indicate the speed of cursor movement. This was added after some initial pilot testing which found that, at times, it was impossible for the user to know if their input was being registered (i.e. if the cursor was moving) as the feedback would not change as the cursor moved through, for example, the first or last third of an item. This speed cue consisted of a short, light 'tap' sound that played at increasing temporal frequency as speed increased. This was designed to sound like the cursor was being rolled across a sawtooth surface. Further piloting found this cue to be beneficial. We considered adding a speed cue to the Positional control feedback, so that the speed of increase/decrease could be represented and the feedback provided would be equal. However, it was concluded that the speed at which the participant would hear the cursor move through the items (via labels and unique tones) would already provide this information, and the same 'tap'/sawtooth design would not work for such variable changes in speed that occur during Positional control.

Before the first trial within a given menu size, the number of items within that menu were communicated via the phrase "[n] items" in synthetic speech, where [n] is $4,6,8$ or 10. The target item was indicated by the phrase "Get [label]" where [label] is one of those from the menus in Figure 3. This phrase was played in the spatialised location around the head relative to its location within the menu. The UMPC screen was blank white.

\section{RESULTS - SESSION 2 \\ Errors}

Comparing the 3 conditions using a Friedman's Test showed a significant effect of Condition on errors $\left(\chi^{2}\right.$ $(2)=62.12, \quad$ p<.001). Wilcoxon pairwise comparisons showed that MPA had significantly higher ER than both SRA $(T=609.0, \mathrm{p}<.001)$ and MRA $(T=704.0, \mathrm{p}<.001)$. Mean ER stood at $2.5 \%$ for SRA, 3.0\% for MRA and $12.8 \%$ for MPA. There was also a significant effect of Menu Size on ER $\left(\chi^{2}(3)=15.26, \mathrm{p}<.01\right)$. Wilcoxon pairwise comparisons showed that the 10 item menu produced significantly more errors than the 4 item menu ( $T=192.50$, $\mathrm{p}<.05)$, the 6 item menu $(T=75.0, \mathrm{p}<.001)$ and the 8 item menu $(T=120.0, \mathrm{p}<.05)$. Overall mean ER for each menu size: $4.5 \%$ for 4 Items, $2 \%$ for 6 Items, $3.9 \%$ for 8 Items and $8.7 \%$ for 10 Items.

\section{Movement Time}

Friedman's Test showed a significant effect of Condition on $\operatorname{MT}\left(\chi^{2}(2)=46.57, \mathrm{p}<.001\right)$. Wilcoxon $T$ showed that each condition had significantly different MT from the other conditions: SRA had lower MT than MRA $(T=117506.50$, $\mathrm{p}<.05)$ and MPA $(T=50710.50, \mathrm{p}<.001)$; MRA had lower MT than MPA $(T=59134.50, \mathrm{p}<.001)$. Mean MT was $3.67 \mathrm{~s}$, $3.96 \mathrm{~s}$ and $5.08 \mathrm{~s}$ for SRA, MRA and MPA respectively.

There was also a significant effect of Menu Size on MT $\left(\chi^{2}\right.$ $(3)=153.02, p<.001)$. Wilcoxon comparisons showed that the MT for all Menu Sizes differed significantly from each other (all $\mathrm{p}<.001)$. MT increased as the Menu Size increased with mean MT of 2.85s, 3.39s, 4.30s and 5.46s for 4, 6, 8 and 10 Item menus respectively.

\section{Number of Crossings/Loops}

As the number of crossings (NC) only applies to Positional control, analysis here was limited to comparing NC across Menu Sizes. A significant effect of Menu Size on NC was found $\left(\chi^{2}(3)=49.627, \mathrm{p}<.001\right)$ with Wilcoxon comparisons showing that all sizes differed from each other significantly: 4 items vs. 6 items and 8 items vs. 10 items $p<.05$; all other pairs $\mathrm{p}<=.001$. Mean $\mathrm{NC}$ for each size was 3.44, 4.96, 9.18 and 13.07 for $4,6,8$ and 10 item menus respectively.

A Wilcoxon comparison of SRA and MRA showed a significant effect of Condition/Mobility on the number of Loops during Rate-based control $(T=1901, \mathrm{p}<.001)$. Mobile selections produced more Loops $($ mean $=0.27)$ per trial than static selections $($ mean $=0.15)$. Friedman's Test also showed a significant effect of Menu Size on Loops $\left(\chi^{2}\right.$ $(3)=25.553, p<.001)$ with Wilcoxon comparisons showing all pairs differed significantly $(\mathrm{p}<.01)$ except for 4 items vs. 6 items and 8 items vs. ten items ( $>$ >.05). Mean number of Loops per trial sat at $0.13,0.14,0.25$ and 0.33 for $4,6,8$ and 10 item menus respectively.

\section{Workload Ratings}

There was a significant effect of Condition on overall subjective workload ratings using Repeated-measures ANOVA $\left(\mathrm{F}_{(2,24)}=7.034, \mathrm{p}<.05\right)$ and Greenhouse-Geisser correction for a significant Mauchly's Sphericity test (chi-square=8.272, $\mathrm{p}<.05$ ). Post hoc Bonferroni pairwise comparisons showed that SRA caused significantly lower overall workload than MPA (p<.05). Mean overall workload ratings were 9.92, 10.79 and 12.71 for SRA, MRA and MPA respectively.

\section{Movement/Control}

Again, we recorded the number of nudges used by participants. The overall average number of nudges across both SRA and MRA was 2.45 nudges per selection. A Wilcoxon pairwise comparison showed a significant effect of Condition/Mobility on the number of nudges ( $T=46446.5$, $\mathrm{p}<.001$ ) with mobile selections producing more nudges per selection $($ mean=2.97) than static selections (mean=1.93). 
Friedman's Test showed that Menu Size also significantly affected the number of nudges $\left(\chi^{2}(3)=49.571, \mathrm{p}<.001\right)$. All menu sizes differed significantly from each other $(\mathrm{p}<=.05)$. Mean number of nudges for each menu size was $1.57,1.81$, 2.50 and 3.07 for 4, 6, 8 and 10 item menus respectively.

\section{DISCUSSION: SESSION 2}

Positional input using audio feedback took longer to make selections and was rated as significantly more mentally \& physically demanding than Rate-based control. Positional input was also significantly more error-prone than Ratebased input. This supports the outcome of session one where the results suggested Rate-based control allows for superior control of cursor movement while mobile. Comparing SRA with MRA showed that mobility increased Movement Time, number of Nudges and Loops which suggests that being mobile had a similar effect on audio interaction as it did on visual interaction, only with a stronger negative effect. However, mobility did not affect accuracy during audio interaction, as SRA and MRA only differed by $0.5 \%$ errors $(2.5 \%$ and $3.0 \%$ respectively). Neither did it affect subjective workload. This suggests that audio-only pressure interaction can reach almost $100 \%$ accuracy even while walking, without sacrificing extra mental/physical demand (compared to sitting audio interaction), albeit at the expense of task time. As in session 1, mobility increased the number of Nudges from 1.93 to 2.97. As is expanded upon below, these numbers are much higher than session one, and the difference between them is also greater.

\section{SESSIONS 1 \& 2 COMPARED: EFFECT OF FEEDBACK}

In this section we compare the Static-Rate (SRV), MobileRate (MRV) and Mobile-Positional (MPV) conditions using visual feedback from session 1 to the audio-only equivalent conditions (SRA, MRA and MPA) from session 2. The Independent Variable for this comparison was Feedback (Visual, Audio).

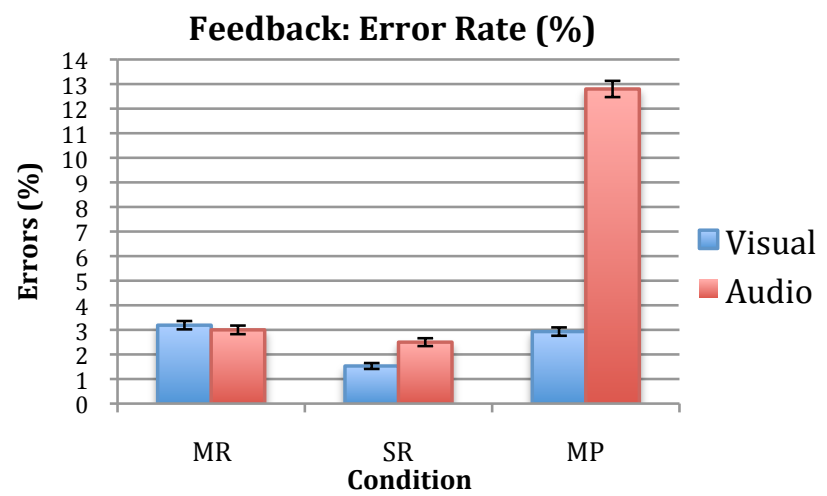

Figure 8: Mean error rates and SD for Mobile-Rate, StaticRate and Mobile-Positional conditions using Visual and Audio feedback.

\section{Errors}

Wilcoxon pairwise comparisons showed a significant difference between SRV and SRA $(T=57.50, \mathrm{p}<.05)$ with the visual condition having lower ER (mean=1.5\%) than the audio condition (mean $=2.5 \%$ ). It was also found that MPV (mean $=2.9 \%$ ) had significantly lower ER than MPA (mean $=12.8 \% ; T=756.50, \mathrm{p}<.001)$. A shown in Figure 8 MRV (mean=3.2\%) and MRA (mean=3.0\%) were not significantly different $(\mathrm{p}>.05)$.

\section{Movement Time}

All three visual conditions in session 1 were significantly faster than the audio equivalents in session $2(\mathrm{p}<.001$; see Figure 9). Mean SRA MT was 1.44s higher than mean SRV; MRA was $1.62 \mathrm{~s}$ slower than mean MRV and MPA was 1.2s slower than MPV (see Figure 9).

\section{Crossings/Loops}

Wilcoxon comparison of MPV and MPA showed a significant effect of Feedback on NC $(T=13028.5, \mathrm{p}<.05)$ with Audio (MPA) selections producing more crossings (mean=7.63) than Visual (MPV) selections (mean=6.25). Wilcoxon comparisons also showed a significant effect of Feedback for both Static (SRV vs. SRA; $T=194.5, \mathrm{p}<.001$ ) and Mobile (MRV vs. MRA; $T=901.5, \mathrm{p}<.001$ ) selections. In both cases audio selections produced more Loops/overshoots than visual selections.

\section{Feedback: Movement Time (s)}

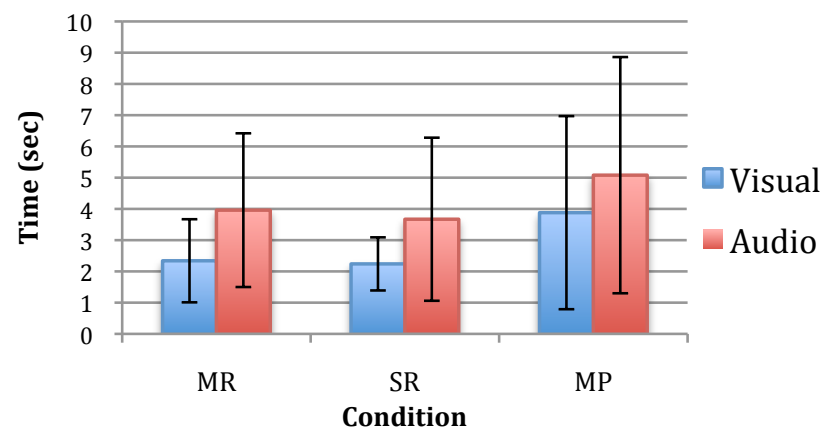

Figure 9: Target selection time and SD for Mobile-Rate, Static-Rate and Mobile-Positional conditions using Visual and Audio feedback.

\section{Workload}

Combining visual and audio conditions into umbrella conditions Mobile-Positional, Mobile-Rate and Static-Rate, a $3 \times 2$ (Condition $x$ Feedback) Repeated-measures ANOVA showed a significant effect of Condition $\left(\mathrm{F}_{(2,24)}=21.254\right.$, $\mathrm{p}<.001)$ and Feedback $\left(\mathrm{F}_{(1,12)}=4.988, \mathrm{p}<.05\right)$ on overall workload rating. Post-hoc Bonferroni tests showed that Mobile-Positional selections (mean=11.91) produced significantly higher workload ratings than both Mobile-Rate (mean=9.25; $\mathrm{p}<.01)$ and Static-Rate selections (mean=8.63; $\mathrm{p}=.001)$. Audio feedback produced higher overall workload ratings $($ mean $=11.14)$ than visual feedback (mean=8.72).

\section{Movement/Control}

Feedback had a significant effect on the number of Nudges for both Static (SRV vs. SRA; $T=735, \mathrm{p}<.001$ ) and Mobile (MRV vs. MRA; $T=2164.5, \mathrm{p}<.001$ ) selections. For both conditions more nudges were used during the audio selections. 


\section{DISCUSSION: SESSION 1 \& 2 COMPARED}

For almost all measures, performance using visual feedback was better than when using audio feedback for all interaction conditions (Mobile, Static, Positional and Rate-based). Therefore audio selections took longer, were more difficult to control and were more mentally/physically demanding. This is perhaps to be expected but there are several interesting results to point out. Firstly, feedback did not affect accuracy during mobile control of linear targeting when using Rate-based control. Mobile control with visual feedback $(\mathrm{ER}=3.2 \%)$ was similarly accurate to mobile control with audio feedback $(E R=3.0 \%)$. Secondly, feedback had a much stronger effect on mobile Positional control than Ratebased control, Positional ER more than quadrupled between visual $(2.9 \%)$ and audio $(12.8 \%)$ conditions.

The number of Nudges was vastly different for visual and audio conditions, increasing from a mean of 0.22 Nudges per selection during visual conditions up to a mean of 2.45 Nudges per selection during audio conditions. Therefore it seems as though participants engaged in searching 'shunt' behaviour much more when only audio feedback was provided. This may be because they still were not familiar enough with the order and layout of items after the familiarisation condition at the start of session 2 . It may also be that they were less confident of their control over the cursor, as the high MT, Loops and Workload, combined with low ER, suggests they had lower levels of control and so found it more difficult to correctly acquire the target item.

\section{GENERAL DISCUSSION \& CONCLUSIONS Control Method}

As was outlined in the introduction and background, a lot of the work that has looked at the use of pressure in HCI interactions has looked at linear targeting using Positional control. During both sessions of this study Rate-based control of linear targeting was found to be faster, similarly or more accurate, and had a lower workload rating than Positional control. This relationship was true while users were both sitting and walking, and with both visual and audio feedback. Even in the stable sitting condition Rate-based control was superior. This echoes to an extent the findings by Shi et al. [13] that Rate-based control allowed for faster and less mentally/physically demanding interaction during shape-translation. Crossan et al. [4] found that Rate-based linear targeting through head-tilting was more accurate than Positional control when the user was walking. From these results it appears that Rate-based control is the optimal control method for pressure-based linear targeting on a mobile device.

Despite this conclusion it is necessary to put our results in context with previous work. The Rate-based Error rates and Movement Times, and the Positional Error rates, are comparable or even superior to those found using Positional control in other work in static scenarios [9, 11, 12]. However the Positional Movement Times and Number of Crossings found here are still higher than other work, even when sitting, suggesting a lower level of control and the Error rate for Positional control while walking was four times as high as when sitting. Although this paper is primarily concerned with the use of pressure for linear targeting, Positional control relates directly to precision of input, and so it gives a clearer indication of how mobility influences pressure input in general, and so deserves attention here.

An explanation for the poorer precision of input may come from the support and stability afforded by the interaction apparatus used. The lowest ER, MT and NC coming from previous work were achieved via either desktop stylus input or FSRs attached to a computer mouse. A stylus grip generally consists of a thumb and two fingers providing opposing and stabilizing forces. Part of the hand and arm are also resting on the table. Those studies using a mouse for linear targeting did not require any $x-y$ movement of the mouse for the interaction so it could remain stationary. Again, the hand and arm would be resting on the table providing stability, with multiple fingers gripping the mouse. These factors provide more stable interactions than in this study, where the user applies force through the thumb or through a thumb-finger pinch while the hands also hold up the device. Although the participant could rest their arms on their knees or the table while sitting, the wrists were unsupported and there was no extra support when mobile. This could make controlling pressure more difficult as it may introduce unintended bodily/hand motion which results in unintended input variation, leading to higher number of Crossings, Loops etc correlated with higher MT, as was found by Crossan et al.[4]. This effect of walking is expanded upon in the next section.

\section{The Influence of Walking}

Walking negatively affected the participants' ability to control pressure. Mobility increased the number of Errors, selection time and Number of Crossings. Measures of control and subjective workload were also higher. However, the effect of mobility was not equal across conditions or variables. As mentioned above, it negatively affected Positional control much more than Rate-based control. Walking significantly decreased overall accuracy during Rate-based control, but, interestingly, only when using visual feedback. It had no effect on overall accuracy when only audio feedback was used: static and mobile non-visual Rate-based interaction was equally accurate. Selection time and overall control degraded under both visual and audio Rate-based walking conditions, however. Mobile interaction was also significantly more mentally/physically demanding. This effect of mobility on accuracy for visual interaction but not audio interaction is intriguing, but may be due to the movement of the device or, more importantly, it's screen. If it was solely the negative influence of walking that was suggested above in relation to Positional control, then the effect should arise for both feedback conditions. During static-visual selections the screen of the device is stationary so cursor movement can be tracked easily. During mobilevisual selections, however, the screen of the device is mov- 
ing, as is the head of the participant, making it potentially harder to track the movement of the cursor. Audio feedback should not have been affected in the same way by bodily motion.

\section{Use of Audio Feedback}

Overall, audio interaction was more error prone (average of $6.1 \%$ errors) than visual interaction (average of $2.5 \%$ ), however error rates remain low and the usefulness of audio feedback for pressure-based interaction while mobile is highly promising. Wilson et al. [16] found overall error rates of $26 \%$ for a very similar audio interaction while seated at a desk and using Positional control. The highest number of errors from both sessions in this study was only $12.8 \%$, and that was for mobile Positional control using audio feedback. Errors for Rate-based control peaked at only $3.2 \%$, during static control (mobile was only $3.0 \%$ ). These error rates are considerably less than those from Wilson et al. even for mobile interaction and comparable or lower to those from studies using seated visual interactions $[3,9,12]$. This paper expanded on those results by looking at whether audio interaction is feasible while the user is walking. Although Movement Time increased, static and mobile audio interaction using Rate-based control was highly accurate. Therefore we had mobile, audio and mobile-audio pressure interaction at similar performance to previous static and visual interactions. This suggests eyesfree mobile pressure interaction is highly feasible.

For all three control conditions using audio feedback in session two (Mobile-Positional, Mobile-Rate, and StaticRate), errors and selection time were higher than the equivalent visual conditions during session one. The difference between visual and audio equivalents was not equal, as audio interaction negatively affected Positional control of pressure much more than Rate-based control. This further highlights the comparatively poorer suitability of Positional control for linear targeting when walking.

\section{Conclusions}

The results from this study have shown that pressure is well suited to menu navigation on mobile devices, with Ratebased control facilitating superior performance over Positional control. Although error rates remain very low for audio and mobile-audio interaction, task time and user workload remain higher than static and visual interaction. The use of larger menu items, or lower numbers of menu items, would be likely to result in faster and easier targeting, resulting in both lower selection time and reduced user workload. Therefore pressure-based menus may be a realistic alternative to touch-based menus with the benefit of enabling 'eyes-free' interaction while on-the-move.

\section{ACKNOWLEDGEMENTS}

This research has been funded by the Industrial Members of MobileVCE (www.mobilevce.com), with additional financial support from EPSRC grant EP/G063427/1.

\section{REFERENCES}

1. Brewster, S. Overcoming the lack of screen space on mobile computers. Personal and Ubiquitous Computing, 2002. 6: p. 188-205.

2. Brewster, S. and M. Hughes. Pressure-Based Text Entry for Mobile Devices. In Proceedings of MobileHCI 2009, Bonn, Germany.

3. Cechanowicz, J., P. Irani, and S. Subramanian. Augmenting the mouse with pressure sensitive input. In Proceedings of CHI 2007, San Jose, California.

4. Crossan, A., M. McGill, S. Brewster, and R. MurraySmith. Head Tilting for Interaction in Mobile Contexts. In Proceedings of MobileHCI 2009, Bonn, Germany.

5. Crossan, A., R. Murray-Smith, and S. Brewster. Gait phase effects in mobile interaction. In CHI 2005 Extended Abstracts, Portland, Oregon.

6. Kildal, J. 3D-Press: Haptic Illusion of Compliance when Pressing on a Rigid Surface. In ICMI 2010, Beijing, China.

7. Marentakis, G. and S. Brewster. Effects of Feedback, Mobility and Index of Difficulty on Deictic Spatial Audio Target Acquisition in the Horizontal Plane. In Proceedings of CHI 2006, Montreal, Quebec.

8. McCallum, D., E. Mak, P. Irani, and S. Subramanian. PressureText: pressure input for mobile phone text entry. In Proceedings of CHI 2009, Boston, MA.

9. Mizobuchi, S., S. Terasaki, T. Keski-Jaskari, J. Nousiainen, M. Ryynanen, and M. Silfverberg. Making an impression: force-controlled pen input for handheld devices. In Proceedings of CHI 2005, Portland, Oregon.

10. Ramos, G. and R. Balakrishnan. Zliding: fluid zooming and sliding for high precision parameter manipulation. In Proceedings of ACM UIST 2005, Seattle, WA.

11. Ramos, G., M. Boulos, and R. Balakrishnan. Pressure widgets. In Proceedings of CHI 2004, Vienna, Austria.

12. Shi, K., P. Irani, S. Gustafson, and S. Subramanian. PressureFish: a method to improve control of discrete pressure-based input. In Proceedings of CHI 2008, Florence, Italy.

13. Shi, K., S. Subramanian, and P. Irani. PressureMove: Pressure Input with Mouse Movement. IFIP INTERACT 2009.

14. Stewart, C., M. Rohs, S. Kratz, and G. Essl. Characteristics of Pressure-Based Input for Mobile Devices. In Proceedings of CHI 2010, Atlanta, Georgia.

15. Tang, H., D.J. Beebe, and A.F. Kramer. A multilevel input system with force-sensitive elements. International Journal of Human-Computer Studies, 2001. 54: p. 495-507.

16. Wilson, G., C. Stewart, and S. Brewster. Pressure-Based Menu Selection for Mobile Devices. In Proceedings of MobileHCI 2010, Lisbon, Portugal. 